\title{
Toxicity test of a dental commercial composite in rats
}

\author{
Santa Ponce-Bravo ${ }^{1}$, Constantino Ledesma-Montes ${ }^{1,2}$, Jorge I. Martínez-Rivera ${ }^{1}$, Israel Morales-Sánchez ${ }^{1}$. \\ ${ }^{1}$ Clinical Oral Pathology Laboratory. Facultad de Odontología, Universidad Nacional Autónoma de México. México, Distrito \\ Federal. MÉXICO. \\ ${ }^{2}$ Cuerpo Académico. Facultad de Odontología, Universidad de Ciencias y Artes de Chiapas. Tuxtla Gutiérrez, Chis. MÉXICO.
}

Ponce-Bravo S, Ledesma-Montes C, Martínez-Rivera JI, Morales-Sánchez I. Toxicity test of a dental commercial composite in rats. J Clin Exp Dent. 2011;3(4):e280-3. http://www.medicinaoral.com/odo/volumenes/v3i4/jcedv3i4p280.pdf

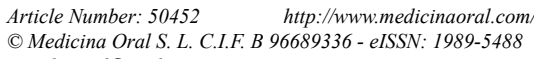

\begin{abstract}
Objective: The aim of this study was to determine the 90-day subchronic toxicity of one triethylene glycol dimethacrylate containing composite (MEDENTAL Light-Cure Composite ${ }^{\mathrm{TM}}$ ) orally administered to rats according to OECD no. 48 guidelines and the requirements specified in the International Organization for Standardization 10993-11.

Study design: The composite was administered orally to Wistar rats during 90 days and they were observed to determine changes in their behavior, eye and skin signs and other attitudes such as aggressiveness, posture, walking and response to handling. After 90 days they were sacrificed to determine blood alterations, special hematological tests were done and histopathological changes in 33 different organs were assessed.

Results: Under the experimental conditions, our results showed that the composite tested in this study did not produce significant changes in clinical behavior of the animals. Microscopic review of the Hematoxilin and Eosin stained slides obtained from 33 analyzed organs showed no abnormal inflammatory or cytological changes and all hematological special tests were within normal limits.

Conclusions: The results of this study show that under our experimental conditions the MEDENTAL Light-Cure Composite $^{\mathrm{TM}}$ does not produce inflammatory or cytological changes suggestive of toxicity.
\end{abstract}

Key Words: Factor XIIIa, radicular cyst, fibrosis, healing. 


\section{Introduction}

Since several years ago, different kinds of esthetic dental materials have been created for Dentistry. Some of them are the reinforced composites; these materials have been accepted as useful restorative dental materials. However, each one has advantages and disadvantages during their use as tooth restoration materials. Physical-chemical properties of these reinforced composites (hardness and compressive strength) are the same as conventional composites $(1,2)$ and studies made on different kinds of dental composites showed that the most important difference among those materials are: their ability to release fluoride, chemical adherence to dentin and compressive strength (1-3). Composites are new materials used in Dentistry with better physical, chemical, biological, radiological and esthetic properties. However, they have some adverse properties as dimensional stress, contraction during polymerization and cellular damage caused by their components (4).

Recently, polyacid compounds have been added to the composites and now they have higher viscosity and strength resistance $(2,4)$. Depending on the trademark, chemical composition of composites is variable and they are made of one organic component Bisphenol glycerolate dimethacrylate (Bis-GMA) or triethylene glycol dimethacrylate (TEDGMA) plus another inorganic component (glass, zirconium, barium, quartz and strontium) and a chemical bond (silano and polyacid groups). The above mentioned materials provide reduced contraction properties in the finally polymerized composite (2-4). Also, it is known that saliva has a deleterious effect decomposing these compounds (6).

Several studies have been published on TEDGMA adverse effects under different experimental and clinical conditions and different parameters were evaluated (7-19); also, some reviews were published $(8,20)$.

It has been demonstrated that TEDGMA induces mitochondrial damage and oxidative stress in human gingival fibroblasts and it causes apoptosis in primary human gingival fibroblasts $(7,9)$. Other study reported that this compound showed a significant enhancement of glucose consumption and lactate production inducing GSH depletion and stimulating glucose-6-phosphate dehydrogenase (G6PDH) and gluthatione reductase (GR) activity (10).

Studying TEDGMA metabolic effects, Engelmann et al (11) showed it increased the phosphomonoester concentration and decreased the phosphodiesters, enhancing the phospholipid turnover. TEDGMA changed the metabolic state of cells indicated by the slight decrease of nucleoside triphosphates and increasing the ratio of nucleoside diphosphates to nucleoside triphosphates. The most remarkable effect of TEDGMA was a nearly complete decline of intracellular glutathione levels.

Kehe et al (12) reported that TEDGMA is toxic to pul- monary cells and pointed on the risk for pulmonary cell damage and it significantly suppressed TNF- $\alpha$ secretion from THP-1 monocytes stimulated with bacterial lipopolysaccharide suggesting that this alteration may influence the biological response of tissues to material in an inflammatory intraoral environment (13).

TEDGMA is toxic to human gingival fibroblasts since it alters the mitochondrial dehydrogenase (MTT) and the lactate dehydrogenase (LDH) activities (14). In another report, Volk et al. (15) reported that TEDGMA depletes intracellular GSH levels at low concentrations suggesting that decrease of GSH is an early reaction which is triggered prior to other cytotoxic alterations. Geurtsen et al. (16) found that TEDGMA has a very high cytotoxicity potential tested in human primary fibroblast cultures. Wataha et al. (20) concluded that TEDGMA containing composites affect or alter cellular functions in Balb/c 3T3 fibroblasts.

The genetic toxicology of TEDGMA was recently reviewed by Schweikl et al. (17) and Geurtsen and Leyhausen (8) wrote on its chemical and biological interactions.

There are few reports on skin reactions associated to TEDGMA: Kanerva (18) reported on the skin allergic reactions to TEDGMA in $8.9 \%$ of the patients tested and Katsuno et al. (19) demonstrated that three TEDGMA containing dentin bonding systems caused contact dermatitis in the skin of guinea pigs. Although, the pathogenic mechanism is not well known $(19,21)$.

For this reason, the aim of this study was to know the 90day oral toxicity of a TEDGMA containing dental composite (MEDENTAL Light-Cure Composite ${ }^{\mathrm{TM}}$ ) orally administered to rats according to OECD no. 48 guidelines and the requirements specified in the ISO 10993-11 $(22,23)$ hypothesizing that no toxic effects exists under our experimental conditions.

\section{Materials and Methods}

Twenty Wistar rats, ten males and ten females, nine weeks old, clinically healthy were used. They were maintained in the Bioterio of the Division de Estudios de Posgrado e Investigación, Facultad de Odontología. Universidad Nacional Autónoma de México. The protocol of this study was approved by the Ethics Committee of our institution.

To perform the current study the recommendations of the OECD no. 48 guidelines and the requirements specified in the ISO 10993-11 were followed $(21,22)$. These documents recommend the use of 20 rats, ten females and ten males. At the beginning of the study, all the animals weighted among $230 \mathrm{~g}$ and $250 \mathrm{~g}$ then, they were weighted each week and reviewed each day for behaviour and clinical signs in eyes, skin, mucous membranes, muck and urine.

The TEDGMA containing composite (Light Cure Com- 
posite ${ }^{\mathrm{TM}}$, MEDENTAL Co., Mexico, Distrito Federal, Mexico) was prepared following the manufacturer instructions. The composite was polymerized (without the bonding system) with a photopolymerizing lamp during 40 seconds, pulverized in a ceramic mortar and pestle and the pulverized powder was stored in sterile Eppendorf tubes. The rats were fed orally during 90 days with $100 \mu \mathrm{g}$ of the pulverized composite everyday in the morning and then, they drank water and food pellets ad libitum. The rats were observed to determine changes in eye signs (irritation and inflammation), skin and mucous membranes (irritation, inflammation, secretions and pruritus), muck, urine (color, consistency, odor), behavior and autonomic activity (aggressiveness, posture, walk and response to handling) and other attitudes according to ISO 10993-11 regulations (23).

In day 90, the rats were anesthetized intra-muscularly with $2 \mathrm{ml}$ of acerpomazine and $1 \mathrm{ml} / \mathrm{Kg}$ of ketamine. Then, by intracardiac puncture $5 \mathrm{ml}$ of blood were obtained from each rat and blood samples were placed in ethylenediaminetetraacetic acid (EDTA) and anticoagulant containing tubes. Later, they were killed by aspiration of chloroform gas and 33 organs: cerebrum, cerebellum, spinal medulla (cervical, mid-thoracic and lumbar), pituitary, thyroid, parathyroid, thymus, esophagus, salivary glands, stomach, small intestine, large intestine, Peyer's patches, liver, pancreas, kidney, adrenal, spleen, heart, trachea, lung, aorta, gonads (ovaries and testicles), prostate, urinary bladder, lymph nodes, peripheral nerves, bone marrow, skin and eyes were immediately immersed in $4 \%$ paraformaldehyde for 24 hours. Specimens were sent to the Clinical and Experimental Pathology Laboratory of our institution and routinely processed to obtain $3 \mu \mathrm{m}$ thick, paraffin embedded, H\&E stained slides. All the slides were observed in a light transmitted microscope (Carl Zeiss, Germany) by two experienced Oral Pathologists.

The hematological studies were as follows: haematocrit, hemoglobin concentration, erythrocyte count, platelet count and total leukocyte count. The laboratory blood tests were: glucose, urea, creatinine, cholesterol, alanine aminotransferase, alkaline phosphatase, total proteins, albumin, potassium and sodium blood-concentrations. These studies were carried out at the Laboratory of Pathology of the Facultad de Veterinaria y Zootecnia, UNAM. Normal animal values were according to the International Species Information System (24).

\section{Results}

After 90 days the rats gained the normal amount of weight. No alterations in the clinical eye signs, skin, mucous membranes, muck, urine, behavior (autonomic activity and attitude). Analysis of $660 \mathrm{H} \& \mathrm{E}$ stained slides of the 33 organs of twenty rats showed no pathological changes in the microscopic architecture of the analyzed organs with no inflammatory, necrotic, degenerative or toxicological changes. Although there were minor changes in the hematological tests, all of them were always within normal limits.

\section{Discussion}

There are several previous studies using purified TEDGMA in various cell cultures (7,9-16). Results from these studies showed that this compound is toxic inhibiting cell metabolism and provoking cell damage. Also, other authors reported on the skin reactions applying TEDGMA in humans and guinea pigs $(18,19)$. These results prompted us to test if the commonly used restorative material MEDENTAL Light-Cure Composite ${ }^{\mathrm{TM}}$ produced clinical or microscopic changes.

During the clinical test we performed to the animals, we did not find changes in the evaluated parameters. Our microscopic review of the samples from the 36 selected organs, evidence of inflammatory or pathologic cellular change was not found. Results of our study strongly suggest that ingestion of the well-polymerized composite we tested (MEDENTAL Light-Cure Composite ${ }^{\mathrm{TM}}$ ) did not produce clinical toxicity, behavioural or cytotoxic changes to the studied organs of the animals. Results of this study suggest that when they are used under the appropriate clinical conditions (as suggested by the manufacturers) and inadvertently ingested, these polymerized composites are clinically safe. It appears that releasing of the "non-polymerized components" of the tested composite during 90 days within the gastrointestinal apparatus of the experimental animals and their absorption to the blood stream did not produce pathologic changes in the studied animals. Results from this study also suggest that in well-polymerized composites their components did not diffuse to the digestive tract and will not produce systemic alterations. It should be pointed out that studies on releasing of these compounds from composites simulating clinical conditions at different days should be done.

The results of this study show that the MEDENTAL Light-Cure Composite ${ }^{\mathrm{TM}}$ does not produce toxicity changes under the employed experimental conditions.

\section{References}

1. Leinfelder KF, Sluder TB, Santos JF, Wall J. Five year clinical evaluation of anterior and posterior restorations of composite resins. Oper Dent. 1980;3:276-81.

2. Gladys S, Van Meerbeek B, Braem M, Lambrechts P, Vanherle G. Comparative physico-mechanical characterization of new hybrid restorative materials with conventional glass ionomer and resin composite restorative materials. J Dent Res. 1997;76:883-94.

3. Braem M, Finger W, Van Dorem VE, Lambrechts P, Vanherle G. Mechanical properties and filler fraction of dental composites. Dent Mater. 1989;5:346-8.

4. Choi KK, Condon JR, Ferracane JL. The effects of adhesive thickness on polymerization contraction stress of composite. J Dent Res. 2000;79:812-7.

5. Hickel R, Dash W, Janda R, Tyas M, Anusavice K. New direct res- 
torative materials. FDI Commission Project. Int Dent J. 1998;48:3-

16.

6. Engelmann J, Janke V, Volk J, Leyhausen G, von Neuhoff N, Schlegelberger B, Geurtsen W. Effects of BisGMA on glutathione metabolism and apoptosis in human gingival fibroblasts in vitro. Biomaterials. 2004;25:4573-80.

7. Lefeuvre M, Amjaad W, Goldberg M, Stanislawski L. TEDGMA induces mitochondrial damage and oxidative stress in human gingival fibroblasts. Biomaterials. 2005;26:5130-7.

8. Geurtsen W, Leyhausen G. Chemical-biological interactions of the resin monomer triethylenglycol-dimetacrylate (TEDGMA). J Dent Res. 2001;80:2046-50.

9. Janke V, von Neuhoff N, Schlegelberger B, Leyhausen G, Geurtsen W. TEDGMA causes apoptosis in primary human gingival fibroblasts. J Dent Res. 2003;82:814-8.

10. Nocca G, De Palma F, Minucci A, De Sole P, Martorana GE, Callá $\mathrm{C}$, et al. Alterations of energy metabolism and glutathione levels of HL-60 cells induced by methacrylates present in composite resins. J Dent. 2007;35:187-94.

11. Engelmann J, Leyhausen G, Leibfritz D, Geurtsen W. Metabolic effects of dental resin components in vitro detected by NMR spectroscopy. J Dent Res. 2001;80:869-75.

12. Kehe K, Reichl FX, Durner J, Walther U, Hickel R, Forth W. Cytotoxicity of dental composite components and mercury compounds in pulmonary cells. Biomaterials. 2001;22:317-22.

13. Noda M, Wataha JC, Lockwood PE, Volkmann KR, Kaga M, Sano H. Sublethal, 2-week exposures of dental material components alter TNF- $\alpha$ secretion of THP-1 monocytes. Dent Mater. 2003;19:101-5.

14. Issa Y, Watts DC, Brunton PA, Waters CM, Duxbury AJ. Resin composite monomers alter MTT and LDH activity of human gingival fibroblasts in vitro. Dent Mater. 2004;20:12-20.

15. Volk J, Engelmann J, Leyhausen G, Geurtsen W. Effects of three resin monomers on the cellular glutathione concentration of cultured human gingival fibroblasts. Dent Mater. 2006;22:499-505.

16. Geurtsen W, Lehman F, Spahl W, Leyhausen G. Cytotoxicity of 35 dental resin composite monomers/additives in permanent 3T3 and three human primary fibroblast cultures. J Biomed Mater Res. 1998;41:474-80.

17. Schweikl H, Spagnuolo G, Schmalz G. Genetic and cellular toxicology of dental resin monomers. J Dent Res. 2006;85:870-7.

18. Kanerva L. Cross-reactions of multifunctional methacrylates and acrylates. Acta Odontol Scand. 2001;59:320-9.

19. Katzuno K, Manabe A, Kurihara A, Itoh K, Hisamitsu H, Wakumoto $\mathrm{S}$, et al. The adverse effect of commercial dentine-bonding systems on the skin of guinea pigs. J Oral Rehabil. 1998;25:180-14.

20. Wataha JC, Rueggeberg FA, Lapp CA, Lewis JB, Lockwood $\mathrm{PE}$, Ergle JW, et al. In vitro toxicity of resin-containing restorative materials after aging in artificial saliva. Clin Oral Investig. 1999;3:144-9.

21. Reichl F, Durner J, Manhart J, Spahl W, Gempel K, Kehe K, et al. Biological clearance of HEMA in guinea pigs. Biomaterials. 2002;23:2135-41.

22. OECD/OCDE. Guideline no. 408: Guideline for the testing of chemicals. Repeated dose 90-day oral toxicity study in rodents. 13th ed. Paris: OCDE; 2002.

23. ISO 10993-11. Biological evaluation of medical devices. Part 11: Tests for systemic toxicity. Geneve: International Organization for Standardization; 1993.

24. Teare JA. Table of Conversion Factors. In: Data Reference Values. International Species Information System Physiological. 2002. Available at: http://www.isis.org/CmsHome/ISIS HomePage. aspx? NRMODE $=$ Published $\&$ NRORIGINALURL $=\% 2 \mathrm{fCMSHO}$ ME\%2f\&NRNODEGUID=\%7b9136F73C-2375-4D4A-AF3F-2F BAFE0CEA5E\%7d\&NRCACHEHINT=NoModifyGuest. 